\title{
Use of Diffusion Tensor Imaging in the Resection of Gliomas
}

\author{
Henry Jung $\cdot$ Aatman Shah $\cdot$ Gordon Li
}

Published online: 23 August 2014

(C) Springer Science + Business Media New York 2014

\begin{abstract}
Standard magnetic resonance imaging techniques provide only anatomic information without information regarding central nervous system connectivity. Overcoming this limitation, diffusion tensor imaging (DTI) can demonstrate the structural integrity and white matter tracts of the brain. In this paper, we will overview the basic principles and techniques of DTI. We will also review the application of DTI in neurosurgery, particularly its application in the resection of gliomas. Tractography provides visualization of the motor tracts and language tracts, which has been corroborated using subcortical mapping/direct stimulation. Furthermore, DTI can also provide information about the arrangement of visual fibers in relation to tumors. Given these advantages, DTI is becoming increasingly used as a surgical planning tool. DTI may provide a safer means for tumor resection by minimizing iatrogenic injury of important functional tracts. Some studies suggest that the use of DTI in tumor surgery may lead to greater Karnofsky performance status scores, greater extent of tumor resection, and increased overall survival.
\end{abstract}

Keywords Diffusion tensor imaging - Magnetic resonance imaging $\cdot$ Neurosurgery $\cdot$ Surgical planning . Glioblastoma · Glioma

This article is part of the Topical Collection on Brain Tumor Surgery.

Henry Jung and Aatman Shah have contributed equally to this study.

H. Jung $(\bowtie) \cdot$ A. Shah · G. Li

Department of Neurosurgery, Stanford Hospital and Clinics, 1201 Welch Rd P309, MSLS, Stanford, CA 94305, USA

e-mail: junger@stanford.edu

\section{Introduction}

Diffusion tensor imaging (DTI) is a powerful technique in the armamentarium of the neurosurgeon. DTI is a form of diffusion-weighted magnetic resonance imaging (MRI) that assesses the directionality of water diffusion. The directional distribution of water diffusivity can be used to construct maps of the microstructure and the white matter tracts of the brain. Because of this capacity to illustrate the white matter tracts of the brain, DTI has been widely utilized in neurosurgery in planning surgical approach and resection.

In this paper, we will overview the basic principles and techniques of DTI. We will then review the uses of DTI in neurosurgery, particularly its application in the resection of gliomas.

\section{Basic Principles}

When water molecules diffuse in all directions with no preferential direction, diffusion is considered "isotropic." In pure water or cerebrospinal fluid, the water molecules diffuse in an isotropic manner. However, when water molecules diffuse in preferential directions, diffusion is called "anisotropic." Anisotropic diffusion is generally seen in white matter tracts because diffusion preferentially moves in parallel to fiber tracts as opposed to perpendicular to fiber tracts.

Diffusion anisotropy is the term used to specify the degree and directionality of water diffusion, and it is a function of the physiochemical properties of the biological tissue and its micro- and macrostructures [1, 2]. DTI assesses diffusion in multiple different directions, and each voxel of the brain can be represented as a diffusion tensor, 


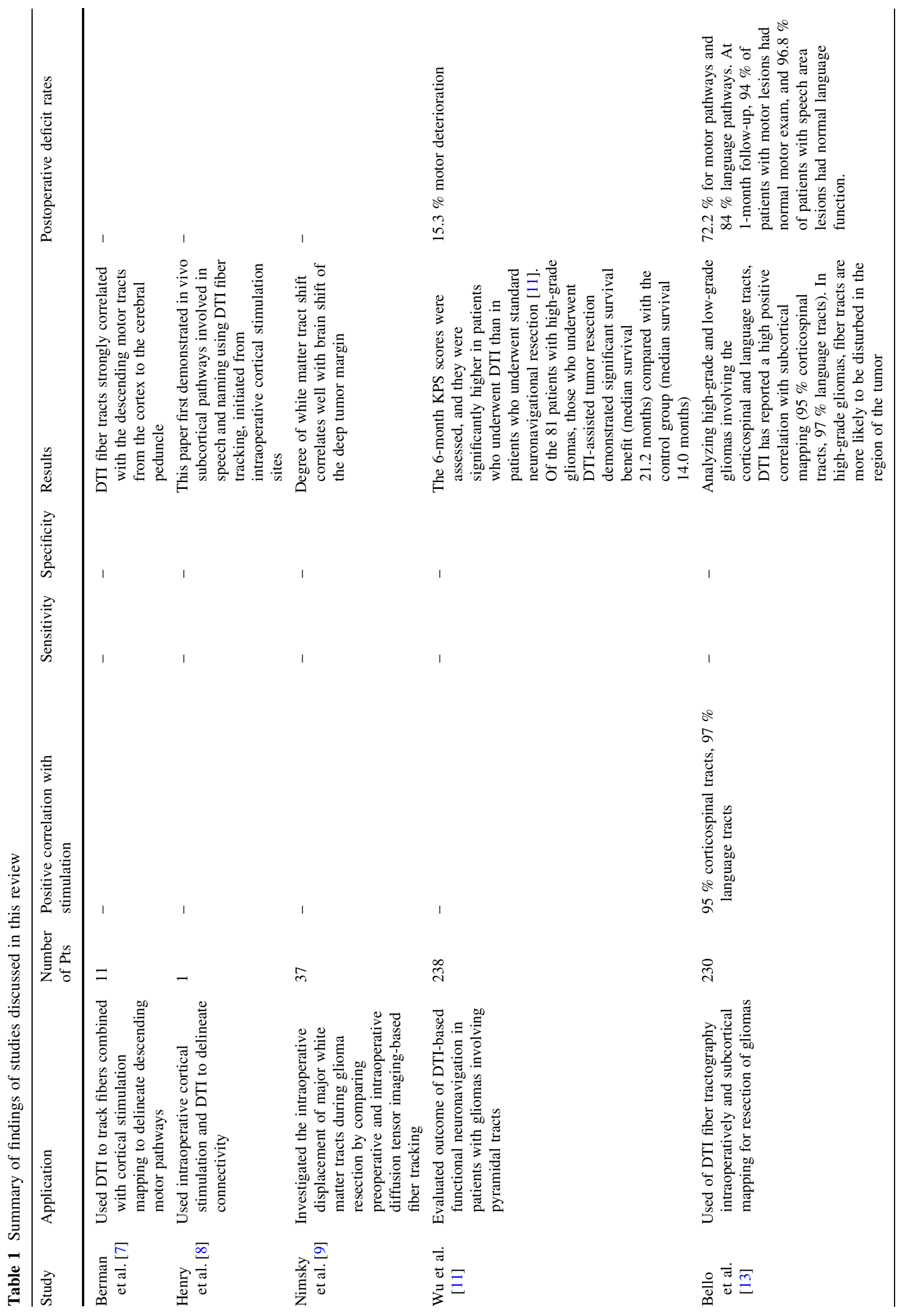




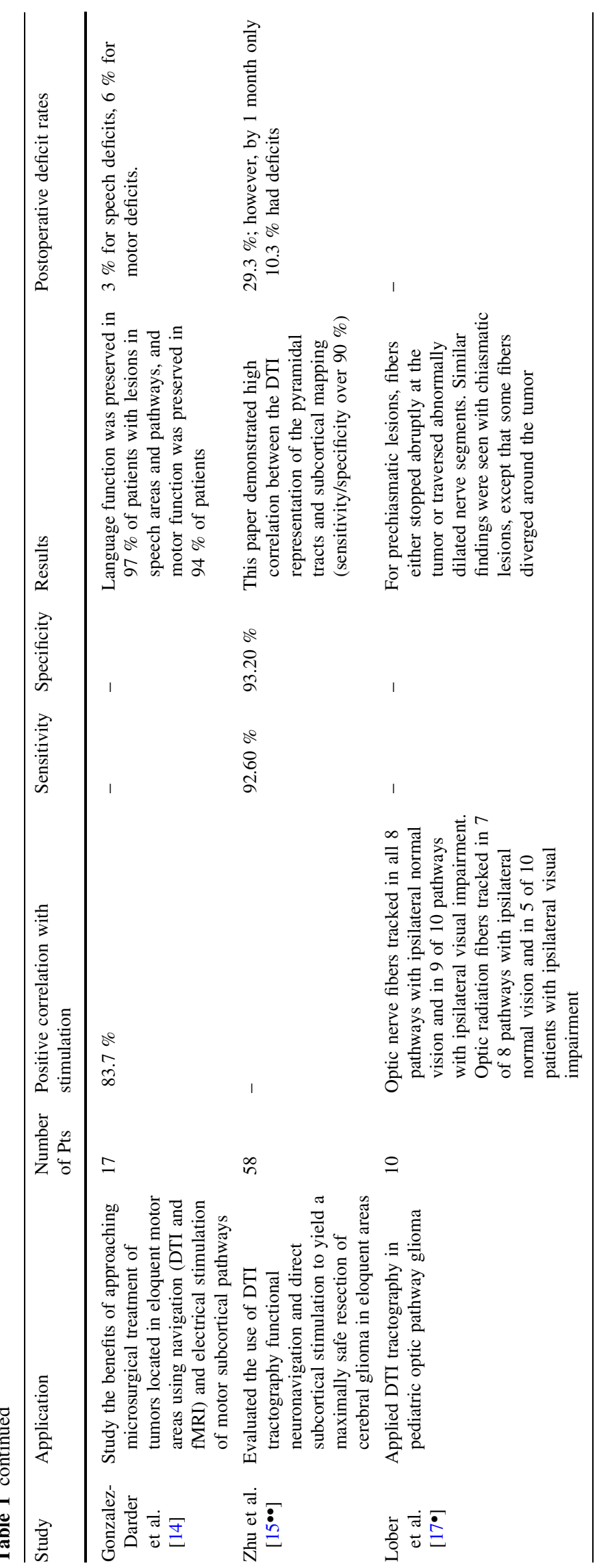


Fig. 1 Diffusion ellipsoid tensors. a Isotropic diffusion can be represented as a sphere with all equal eigenvalues. When diffusion is isotropic, fractional anisotropy (FA) is zero. $\mathbf{b}$ In the brain and especially in white matter tracts, diffusion is anisotropic. The tensor in such a case can be represented as an ellipsoid. $F A>0$

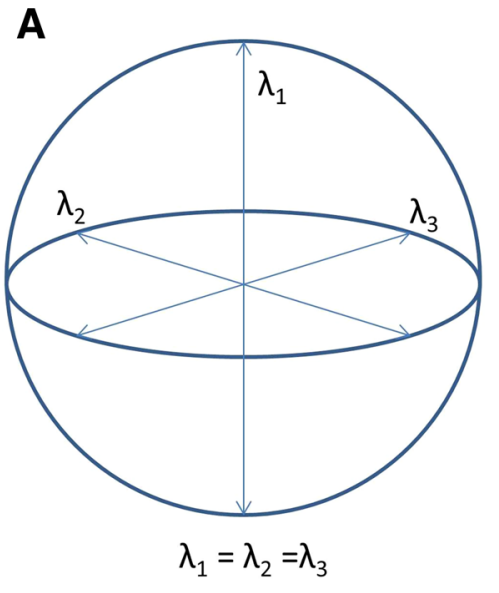

B

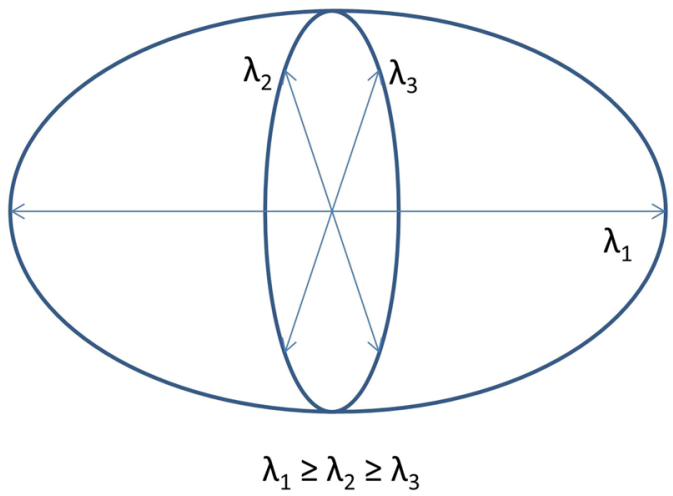

which can be thought of as a multidimensional diffusion vector. The diffusion tensor is characterized by three eigenvalues $\left(\lambda_{1} \geq \lambda_{2} \geq \lambda_{3}\right)$, which represent magnitude of the diffusivities, as well as three eigenvectors $\left(v_{1}, v_{2}, v_{3}\right)$, which represent the direction of the diffusion of each voxel. These three eigenvectors are all orthogonal with one another. By convention, the diffusion along the principal axis (direction of maximum diffusion) is along direction of $\mathrm{v}_{1}$ with magnitude $\lambda_{1}$.

In isotropic diffusion, the tensor has diffusion coefficients that are equal in all directions $\left(\lambda_{1}=\lambda_{2}=\lambda_{3}\right)$, and hence, can be visualized as a sphere (see Fig 1a). In contrast, when the diffusion coefficients are different magnitudes, the tensor can be visualized as an elongated ellipsoid (see Fig 1b).

Common metrics calculated in DTI include mean diffusivity and fractional anisotropy. Mean diffusivity is the directionally averaged diffusivity of water and can be calculated as the mean of all eigenvalues [3•]. The degree of diffusion anisotropy is given by the fractional anisotropy, which provides a normalized value of a tensor's anisotropy; zero represents complete isotropy and one represents complete anisotropy. Using fractional anisotropy, anatomic maps of the fiber tracts can be created. The directionality is usually presented in three standard colors: green (anteroposterior, e.g., association fibers), red (transverse, e.g., commissural fibers), and blue (craniocaudal, e.g., corticospinal tract).

\section{Data Acquisition}

Images are generally acquired in a 3T MRI with a standard head coil. Images are obtained using a single-shot echoplanar imaging (EPI) sequence. Single-shot EPI is commonly used as it has a shorter acquisition time, superior signal-to-noise ratio, and less susceptibility-related distortion than multishot EPI. Cardiac gating, cerebral spinal fluid suppression, and navigator echo correction are used to reduce motion-related artifacts at the expense of prolonged imaging time [4•]. Acquisition times are usually less than 5 min [4•]. Six images are obtained by applying a diffusion-weighted gradient in at least six different directions, and the elements of the symmetric diffusion tensor $\left(D_{\mathrm{xx}}\right.$, $D_{\mathrm{yy}}, D_{\mathrm{zz}}, D_{\mathrm{xy}}, D_{\mathrm{xz}}$, and $\left.D_{\mathrm{yz}}\right)$ can be obtained from the data acquired. The three eigenvalues and eigenvectors can be calculated from these tensor elements.

\section{Techniques}

The tensors of the cerebral white matter can be used to reconstruct the three-dimensional fiber orientation from voxel to voxel, and this is the basis of fiber tractography. The two predominant techniques used to generate fiber trajectories are the deterministic line propagation method and the probabilistic method.

The most common method is the line propagation technique $[4 \cdot 5 \bullet]$. With this technique, a region of interest is selected, and tracking is started voxel by voxel in which a line is propagated in both an anterograde and retrograde manner along the principal axis. Tracking terminates when the fiber tract reaches a voxel with a fractional anisotropy below a given low threshold value (usually between 0.1 and 0.2). One limitation of the line propagation technique is that it can only create one trajectory per seed voxel.

Another technique in fiber tractography is the probabilistic method $[4 \cdot, 5 \bullet]$. This approach creates a probabilistic map of "likely" tracts, and thus, is able to generate multiple pathways from each seed voxel as it computes the probability of a dominant trajectory passing through a region of interest. This probabilistic approach, therefore, may highlight other connectivities between brain regions that were not identified via the line propagation technique either because of nondominancy or uncertainty. The probabilistic approach does have limitations in neurosurgical planning 


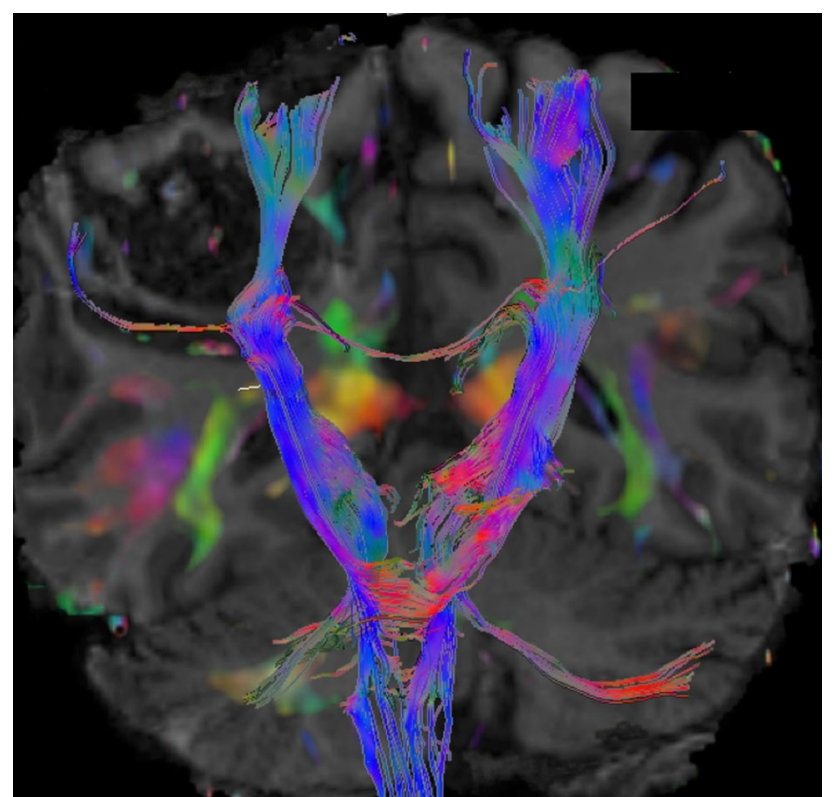

Fig. 2 Diffusion tensor imaging demonstrating the corticospinal tracts

$[5 \cdot, 6]$. As this method is computationally complex, it is not suitable for interactive use. Also, as this approach is not creating discrete geometric pathways, the tracts are harder to visualize and judgment is required to determine anatomic relevance. Further clinical validation is needed for tractography as a surgical planning tool.

\section{The Application of DTI in Glioma Resection}

Standard MRI techniques provide only anatomic information without information regarding central nervous system connectivity. The ability to visualize important white matter tracts facilitates the surgical approach to resection [3•]. Berman et al. [7] used both DTI and direct cortical stimulation in eleven patients who underwent glioma resection. They demonstrated that the DTI fiber tracts strongly correlated with the descending motor tracts from the cortex to the cerebral peduncle. Figure 2 demonstrates the corticospinal tracts. Figure 3 demonstrates a large oligodendroglioma in the left frontal lobe displacing the motor cortex and its tracts medially and posteriorly.

DTI has also shown in vivo the white matter tracts associated with speech, corroborated by cortical stimulation during resection of a glioma [8]. If a tumor is in the vicinity of a suspected language area, such as the left parietal area, a DTI can reveal the anatomic relationship of a tumor with important language tracts. Figure 4 shows a left parietal glial tumor, measuring $2 \mathrm{~cm}$ with surrounding edema. The DTI reveals the anatomic relation between the tumor and the arcuate fasciculus and inferior longitudinal fasciculus, which are depicted with green arrows. Figure 5 shows a cavernous malformation in relation to the arcuate fasciculus (shown in red) and the optic radiations (shown in green). The cavernous malformation was resected, while the patient was awake. During the more medial surgical approach while in proximity to the optic radiations, the patient reported seeing flashes of light. Figure 6 demonstrates how a glioblastoma tumor in the right frontal lobe distorts the corpus callosum.

In 2005, one study looked at the pre- and intraoperative use of DTI for supratentorial glioma surgery [9], and the shift of white matter tracts was analyzed. This study was one of the largest cohorts to undergo glioma resection aided by DTI without the use of direct cortical stimulation [9]. The study showed that the degree of white matter tract shift correlated well with brain shift of the deep tumor margin, as seen previously [10].

$\mathrm{Wu}$ et al. [11] investigated the impact of DTI versus standard neuronavigation in treating gliomas involving the pyramidal tracts. The study had a series of 238 patients, 118 of whom underwent DTI fiber tracking integrated into neuronavigation during resection, and 120 of whom underwent standard MRI and standard neuronavigation. Follow-ups lasted up to 50 months. The 6-month Karnofsky performance status (KPS) scores were assessed, and they were significantly higher in patients who underwent DTI than in patients who underwent standard neuronavigational resection [11]. Of the 81 patients with high-grade gliomas, those who underwent DTI-assisted tumor resection demonstrated significant survival benefit (median survival 21.2 months) compared with the control group (median survival 14.0 months) [11]. For a subset of 50 patients with glioblastoma/gliosarcoma, there was a significant difference in median survival in the DTI group (19.3 months) compared with the control group (11.2 months). This represents a $53.6 \%$ reduction in the risk of death [12]. Moreover, the overall gross total resection rates in the DTI group (72\%) were significantly higher than the gross total resection rates in the control group $(51.7 \%)$. This study demonstrates benefits in increasing overall survival, extent of resection, and 6-month KPS score when DTI-assisted treatment is used for high-grade gliomas. However, the study does have limitations: (1) Progression-free survival rates and neurological outcome for resection of low-grade gliomas were not demonstrated. (2) The baseline KPS scores for patients with high-grade gliomas were not provided. High preoperative KPS score is correlated with survival. Nevertheless, the study corroborates the use of DTI in the surgical planning of gliomas. 

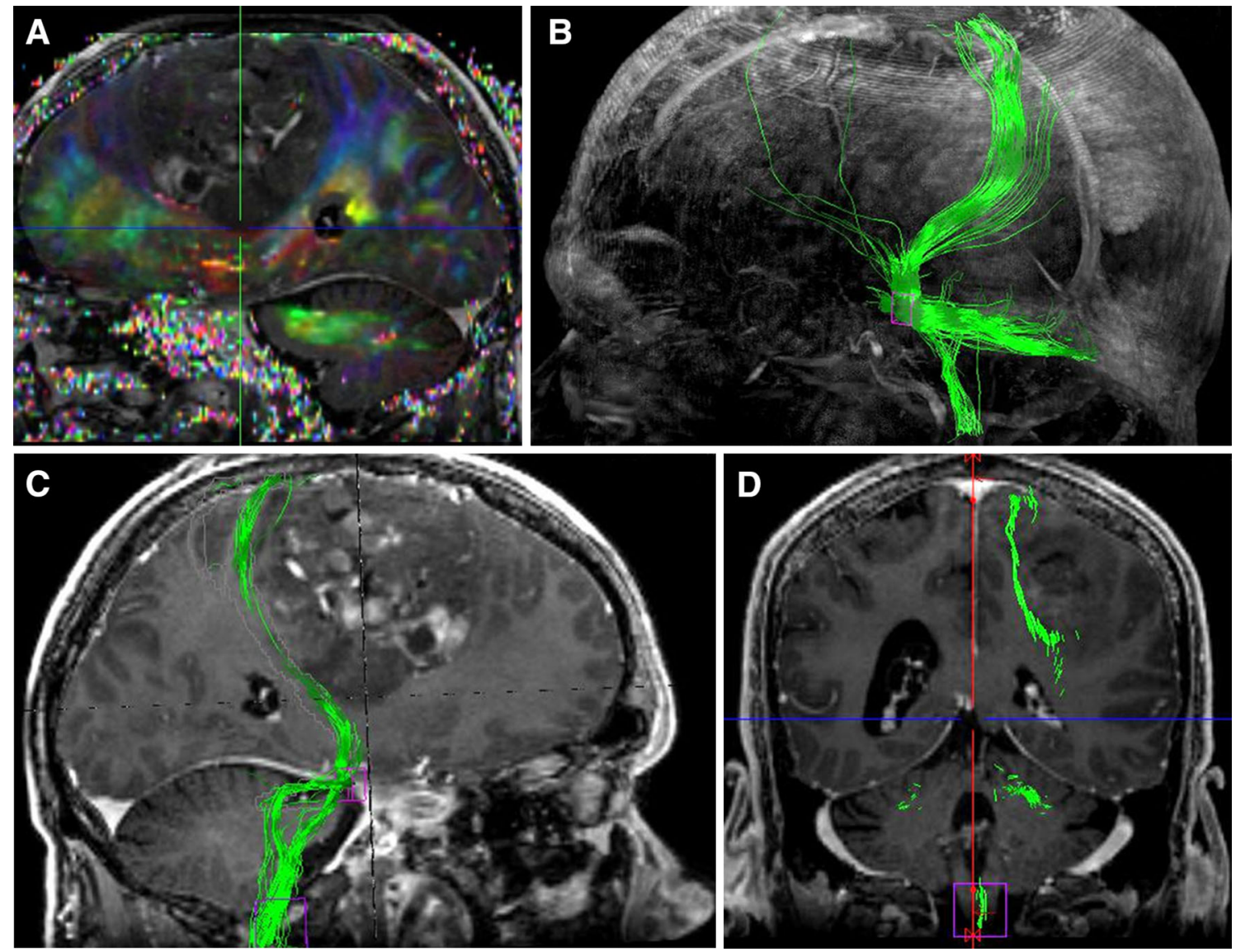

Fig. 3 Left frontal oligodendroglioma. a, b, c Sagittal views of DTI image showing the tumor displacing the motor tracts posteriorly and medially. d Coronal DTI image

\section{DTI Correlation with Cortical and Subcortical Mapping in Glioma Resection}

DTI provides anatomic information, and cortical stimulation provides functional information. This difference between structure and function is particularly salient when resecting language area tumors, as the anatomic tracts can be more expansive than their functionally relevant counterparts [13]. Incorporating anatomic and functional information is essential. Multimodal analysis may involve cortical/subcortical stimulation in addition to functional MRI and DTI for lesion localization and resection. One study utilized this multimodal method for evaluating 17 patients who underwent glioma resection in motor areas [14]. There was significant correlation between cortical areas tested with stimulation and the density of motor fibers seen on DTI. With standard anatomic analysis, the central sulcus and precentral gyrus were recognized in 11 of 17 cases. Functional MRI without direct stimulation was not useful in recognizing target areas because of signal distortion caused by the tumor. However, when DTI was used in conjunction with the above, the central sulcus and precentral gyrus were identifiable in all cases [14].
Studies correlating DTI of language tracts with cortical/ subcortical stimulation have shown varying results. One group evaluated low-grade gliomas and dysplasia involving language tracts and reported only an $82 \%$ positive correlation of fiber tractography with subcortical stimulation. This study suggests that an inability to visualize fibers with DTI does not necessarily rule out a fiber tract in that region. When an area is invaded by a glioma, failure of fiber tracking is most likely caused by decreased fractional anisotropy values. In contrast, another study, analyzing high-grade and low-grade gliomas involving the corticospinal and language tracts, reported a positive correlation (95\% corticospinal tracts, $97 \%$ language tracts) [13]. This study evaluated 230 patients with gliomas who underwent DTI and subcortical mapping of the corticospinal and language tracts [13]. Using both fiber tractography and direct stimulation, language function was preserved in $97 \%$ of patients who had lesions in speech function areas and pathways, and preserved motor function in $94 \%$ of patients [13]. In high-grade gliomas, fiber tracts are more likely to be disturbed in the region of the tumor. Altering thresholds, such as decreasing the minimum fractional anisotropy or increasing the maximum angle, may improve fiber visualization [13]. 


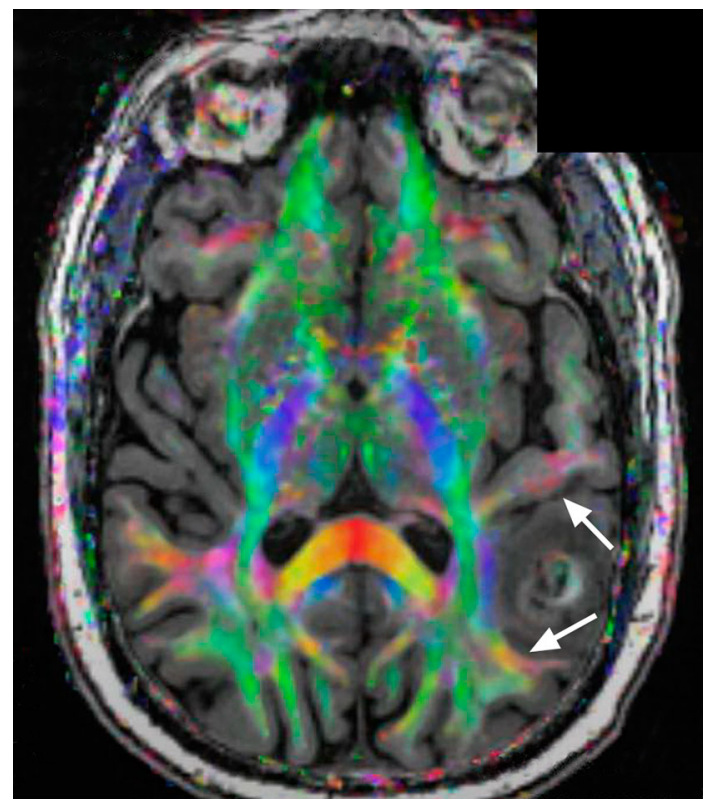

Fig. 4 Brain tumor in eloquent areas. The DTI image reveals the anatomic relation between the tumor and the arcuate fasciculus and inferior longitudinal fasciculus, which are both depicted with white arrows

Recent studies have corroborated the relation between DTI and subcortical mapping. Zhu et al. [15••] conducted a study of 58 patients with gliomas near the pyramidal tract and found a high correlation between the DTI representation of the pyramidal tracts and subcortical mapping (sensitivity/specificity over $90 \%$ ). Another group looked at 190 patients who underwent resection of low-grade gliomas [16]. While all underwent subcortical stimulation, 117 patients underwent resection along with DTI and functional MRI. The extent of resection was significantly greater in the patients who had undergone DTI and functional MRI-assisted neuronavigation. While subcortical stimulation helps to avoid iatrogenic injury to important tracts, it cannot prevent potential vascular injury to the periphery of those tracts, as DTI can.
Although cortical and subcortical gliomas have been the emphasis of the majority of studies analyzing DTI in tumor resection, this imaging has also been utilized in resecting gliomas adjacent to other important neural structures. Studies have demonstrated the value of DTI of lesions near the optic pathway and associated radiations. Lober et al. [17•] visualized the optic pathway fibers in ten patients with optic gliomas. Furthermore, they demonstrated that optic pathway tractography provides new information about the arrangement of visual fibers in relation to tumors, which can be used in surgical planning. Others have also used DTI for the visualization of the optic radiations [18]. Table 1 summarizes the findings from papers discussed in this review.

Setzer et al. [19] assessed the viability of the use of DTI in patients with intramedullary spinal cord tumors. The tumors were classified as resectable or unresectable based on the fiber course proximity to the tumor. In all of the cases where fibers did not enter the tumor, the tumor was successfully resected; in the cases where most of the fibers either entered or were destroyed by the tumor, five of six tumors were deemed unresectable.

\section{Challenges of DTI}

Neuronavigation has immensely improved the ability of neurosurgeons to operate more safely within areas of the brain, and DTI has been shown to be a valuable technique in delineating important neural tracts. DTI is progressively becoming more widely used at many centers. However, there remain some challenges with the use of DTI. Diffusion-weighted imaging is inherently a noise-sensitive and artifact-prone MRI technique. Another challenge is brain shift, which is challenging to account for. DTI mapping has been shown to be reliable, but brain shift occurs after the dura mater is opened. Some centers use direct stimulation, ultrasound, or intraoperative MRI to account for this shift [3•]. Furthermore, tracts are often disturbed by the lesion.
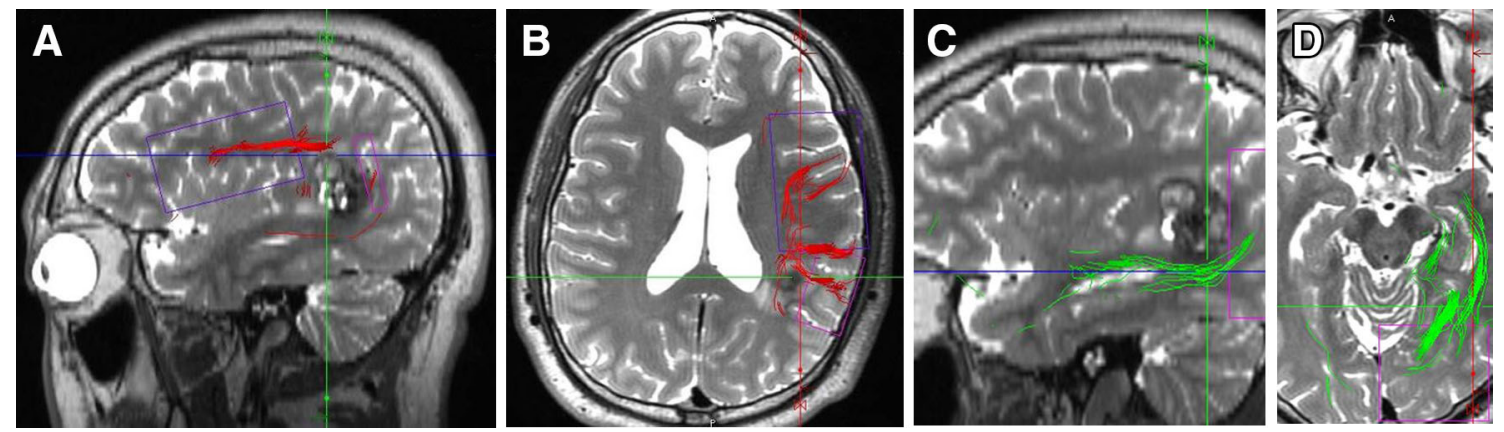

Fig. 5 Left parietal cavernous malformation. a Sagittal DTI image shows relation of arcuate fasciculus with cavernous malformation. b Axial DTI image showing arcuate fasciculus c Sagittal DTI image showing optic radiations. d Axial DTI image showing optic radiations 

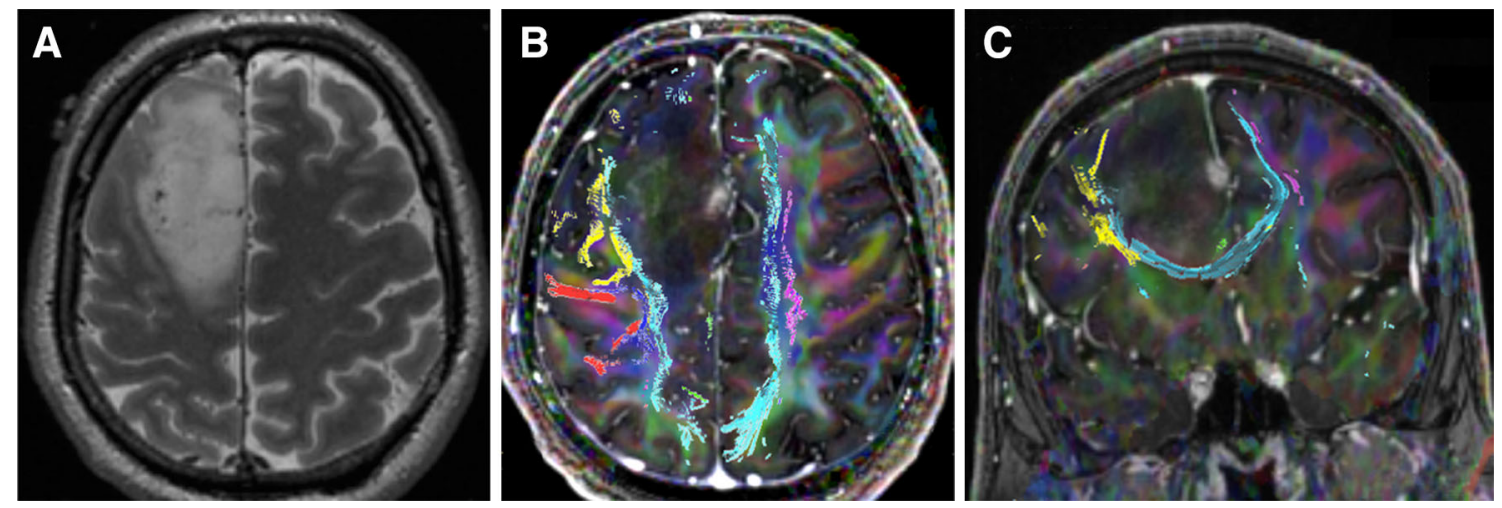

Fig. 6 Right frontal glioblastoma. a Axial T2 scan of tumor demonstrating a large right frontal tumor with edema. b Axial DTI image showing the tumor laterally displacing the corpus callosum. c Coronal DTI image showing lateral displacement of the corpus callosum

Gliomas can distort fiber tracts through penetration or vasogenic edema, resulting in a lowering of the anisotropy value. The complex nature of gliomas has a confounding effect upon DTI and is an intrinsic problem that supports the use of multimodal adjunct technologies for resection. Reproducibility of DTI metrics is a concerning limitation in clinical application. DTI metrics may vary when different scanners are used, even when the same scanner models are utilized [20]. Longitudinal cohorts may also be subject to varying DTI metrics if the scanner software is upgraded [21].

DTI in the peritumoral region is limited by many factors, such as decreased fractional anisotropy with failure of tractography, mass effect, edema, infiltration by tumor leading to spurious fibers, and fiber distortion obscuring tract anatomy [5•]. Tractography should not be used as the only technique for preoperative planning. Directional color-coded fractional anisotropy maps and MRI should be used along with tractography to improve visualization of distorted, infiltrated tracts [22]. Furthermore, the widespread use of DTI in neurosurgical navigation is still limited by the lack of standardized methods. Bürgel et al. [23] evaluated corticospinal tract demarcation created by four separate fiber tracking tools and found that the results were not comparable. Caution should be used when tractography is used for operative planning, especially when fiber tracts are distorted or invaded.

\section{Conclusion}

DTI has empowered the neurosurgeon by better delineating the functionally important tracts of the brain and spinal cord. DTI is now being routinely performed for many brain tumors, especially for tumors within or near the motor and language areas. Although not discussed in this review, DTI has had promising diagnostic capabilities in epilepsy, traumatic brain injury, multiple sclerosis, Alzheimer's disease, and ischemic stroke. DTI, especially DTI tractography, should be applied with caution, and one should be cognizant of its limitations. Thus far, DTI has been limited to corroboration of known tracts. With further research, the DTI technique continues to be optimized. It is already being commonly used as a surgical planning tool, and it may provide a safer means in tumor resection by minimizing iatrogenic injury of important functional tracts. Some studies suggest that the use of DTI in tumor surgery may lead to less morbidity, greater extent of tumor resection, and increased overall survival. Greater knowledge of the functional and anatomic relations between a brain tumor and its eloquent and subcortical surrounding structures will serve to enhance the safety and efficacy of brain tumor surgery.

Acknowledgments The authors would like to thank Cheryl Christensen for editing this manuscript.

\section{Compliance with Ethics Guidelines}

Conflict of Interest Henry Jung, Aatman Shah, and Gordon Li declare no conflict of interest.

Human and Animal Rights and Informed Consent This article does not contain any studies with human or animal subjects performed by the authors.

\section{References}

Papers of particular interest, published recently, have been highlighted as:

- Of importance

- Of major importance

1. Pierpaoli C, Jezzard P, Basser PJ, Barnett A, Di Chiro G. Diffusion tensor MR imaging of the human brain. Radiology. 1996;201(3):637-48. 
2. Kingsley P. Introduction to diffusion tensor imaging mathematics: Part I tensors, rotations, and eigenvectors. Concepts Magn Reson A. 2006;28A(2):101-22.

3. - Abdullah KG, Lubelski D, Nucifora PG, Brem S. Use of diffusion tensor imaging in glioma resection. Neurosurg focus. 2013;34(4):E1. Important article discussing the scientific principles of DTI includes a primer on the technology and image acquisition. Furthermore, it outlines DTI as a tool for glioma resection highlighting important clinical studies on its application.

4. - Ahn S, Lee SK. Diffusion tensor imaging: exploring the motor networks and clinical applications. Korean $\mathbf{J}$ Radiol. 2011;12(6):651-661. This paper provides a nice review of the basic principles of diffusion weighted imaging and its application in a variety of neurological pathologies.

5. - Lerner A, Mogensen MA, Kim PE, Shiroishi MS, Hwang DH, Law M. Clinical applications of diffusion tensor imaging. World neurosurg. 2013. This paper is an excellent review of applications of diffusion tensor imaging neurological pathologies, including spinal cord pathologies.

6. Behrens TE, Johansen-Berg $\mathrm{H}$, Woolrich MW, Smith SM, Wheeler-Kingshott CA, Boulby PA, Barker GJ, Sillery EL, Sheehan K, Ciccarelli O, et al. Non-invasive mapping of connections between human thalamus and cortex using diffusion imaging. Nat Neurosci. 2003;6(7):750-7.

7. Berman JI, Berger MS, Mukherjee P, Henry RG. Diffusion-tensor imaging-guided tracking of fibers of the pyramidal tract combined with intraoperative cortical stimulation mapping in patients with gliomas. J Neurosurg. 2004;101(1):66-72.

8. Henry RG, Berman JI, Nagarajan SS, Mukherjee P, Berger MS. Subcortical pathways serving cortical language sites: initial experience with diffusion tensor imaging fiber tracking combined with intraoperative language mapping. NeuroImage. 2004;21(2): 616-22.

9. Nimsky C, Ganslandt O, Hastreiter P, Wang R, Benner T, Sorensen AG, Fahlbusch R. Preoperative and intraoperative diffusion tensor imaging-based fiber tracking in glioma surgery. Neurosurgery. 2005;56(1):130-7 discussion 138.

10. Dorward NL, Alberti O, Velani B, Gerritsen FA, Harkness WF, Kitchen ND, Thomas DG. Postimaging brain distortion: magnitude, correlates, and impact on neuronavigation. J Neurosurg. 1998;88(4):656-62.

11. Wu JS, Zhou LF, Tang WJ, Mao Y, Hu J, Song YY, Hong XN, $\mathrm{Du}$ GH. Clinical evaluation and follow-up outcome of diffusion tensor imaging-based functional neuronavigation: a prospective, controlled study in patients with gliomas involving pyramidal tracts. Neurosurgery. 2007;61(5):935-48 discussion 948-939.

12. Yogarajah M, Focke NK, Bonelli SB, Thompson P, Vollmar C, McEvoy AW, Alexander DC, Symms MR, Koepp MJ, Duncan JS. The structural plasticity of white matter networks following anterior temporal lobe resection. Brain. 2010;133(Pt 8):2348-64.

13. Bello L, Castellano A, Fava E, Casaceli G, Riva M, Scotti G, Gaini SM, Falini A. Intraoperative use of diffusion tensor imaging fiber tractography and subcortical mapping for resection of gliomas: technical considerations. Neurosurg Focus. 2010;28(2):E6.
14. Gonzalez-Darder JM, Gonzalez-Lopez P, Talamantes F, Quilis V, Cortes V, Garcia-March G, Roldan P. Multimodal navigation in the functional microsurgical resection of intrinsic brain tumors located in eloquent motor areas: role of tractography. Neurosurg Focus. 2010;28(2):E5.

15. •• Zhu FP, Wu JS, Song YY, Yao CJ, Zhuang DX, Xu G, Tang WJ, Qin ZY, Mao Y, Zhou LF. Clinical application of motor pathway mapping using diffusion tensor imaging tractography and intraoperative direct subcortical stimulation in cerebral glioma surgery: a prospective cohort study. Neurosurgery. 2012;71(6):1170-1183; discussion 1183-1174. Important cohort study prospectively evaluating the viability of DTI tractography and direct subcortical stimulation in resection of cerebral glioma in eloquent areas. These data are important for evaluating DTI usage.

16. Ius T, Isola M, Budai R, Pauletto G, Tomasino B, Fadiga L, Skrap M. Low-grade glioma surgery in eloquent areas: volumetric analysis of extent of resection and its impact on overall survival. A single-institution experience in 190 patients: clinical article. J Neurosurg. 2012;117(6):1039-52.

17. - Lober RM, Guzman R, Cheshier SH, Fredrick DR, Edwards MS, Yeom KW. Application of diffusion tensor tractography in pediatric optic pathway glioma. J Neurosurg Pediatr. 2012;10(4):273-280. Important study assessing the role of DTI tractography in analyzing the location of visual pathway fibers in the presence of a tumor. DTI provided further information about fiber arrangement in relation to the tumor not evident in MRI. This information is important in assessing the viability and usage of DTI.

18. Sun GC, Chen XL, Zhao Y, Wang F, Hou BK, Wang YB, Song $\mathrm{ZJ}$, Wang $\mathrm{D}, \mathrm{Xu} \mathrm{BN}$. Intraoperative high-field magnetic resonance imaging combined with fiber tract neuronavigation-guided resection of cerebral lesions involving optic radiation. Neurosurgery. 2011;69(5):1070-84 discussion 1084.

19. Setzer M, Murtagh RD, Murtagh FR, Eleraky M, Jain S, Marquardt G, Seifert V, Vrionis FD. Diffusion tensor imaging tractography in patients with intramedullary tumors: comparison with intraoperative findings and value for prediction of tumor resectability. J Neurosurg Spine. 2010;13(3):371-80.

20. Takao H, Hayashi N, Ohtomo K. Effect of scanner in asymmetry studies using diffusion tensor imaging. NeuroImage. 2011;54(2): 1053-62.

21. Takao H, Hayashi N, Kabasawa H, Ohtomo K. Effect of scanner in longitudinal diffusion tensor imaging studies. Hum Brain Mapp. 2012;33(2):466-77.

22. Leclercq D, Delmaire C, de Champfleur NM, Chiras J, Lehericy S. Diffusion tractography: methods, validation and applications in patients with neurosurgical lesions. Neurosurg Clin North Am. 2011;22(2):253-68 ix.

23. Burgel U, Madler B, Honey CR, Thron A, Gilsbach J, Coenen VA. Fiber tracking with distinct software tools results in a clear diversity in anatomical fiber tract portrayal. Central Eur neurosurg. 2009;70(1):27-35. 